EPJ Web of Conferences 41, 06008 (2013)

DOI: $10.1051 /$ epjconf/20134106008

C) Owned by the authors, published by EDP Sciences, 2013

\title{
Hydrophobic hydration of globular proteins studied with 2D- IR spectroscopy
}

\author{
J. T. King, E. J. Arthur, C. L. Brooks III and K. J. Kubarych \\ Department of Chemistry, University of Michigan, Ann Arbor, MI 48109, USA
}

\begin{abstract}
Ultrafast 2D-IR spectroscopy of a CO ligand bound to a copper active site of an artificial metalloenzyme is used as a sensitive probe of both the flexibility and the electrostatic environment of the engineered catalyst.
\end{abstract}

\section{Introduction}

In recent years the view of water has changed from being an inert medium for biological processes to being an active component of cellular functions, from DNA replication to enzyme catalysis.[1] In particular, the interaction between hydrophobic material and surrounding water can have significant implications on the properties of hydrating water.[2] The diverse amino acids that comprise proteins lead to folded globular proteins with heterogeneous surfaces ranging from structured, hydrophobic regions to random coil segments containing mostly hydrophilic residues. The heterogeneous nature of the protein surface naturally endows the

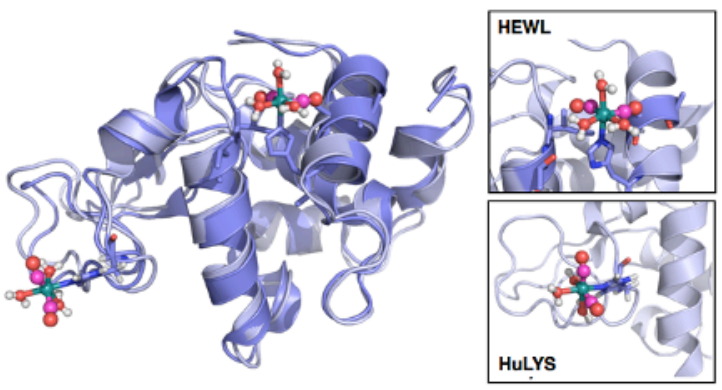

Fig. 1 Structure of HEWL-RC and HuLys-RC superimposed, highlighting the high degree of structural similarity between the two proteins. The insets show the local binding areas of the rutherium-dicarbonyl probe on each protein.

hydration water with a range of dynamical properties as well. Here we study two homologous proteins, hen egg white lysozyme (HEWL) and human lysozyme (HuLys), vibrational labeled with a strong ruthenium-carbonyl probe (labeled proteins referred to as HEWL-RC and HuLys-RC). The two proteins provide an excellent opportunity to compare hydration dynamics near a highly structured region of the protein found at the labeling site of HEWL-RC, and a random coil portion of a protein near the labeling site of HuLys-RC (Figure 1).

\section{Constrained hydration dynamics}

Hydrophobic hydration is often associated with a dynamically constrained hydration water. The vibrational lifetimes of coupled $\mathrm{CO}$ oscillators in aqueous environments are characteristically fast, and the sub-5 ps relaxation can be used as an effective water sensor (contrasted to the 30-50 ps 
lifetimes observed in alcohol solvents). Furthermore, the relaxation is $30 \%$ faster in $\mathrm{H} 2 \mathrm{O}$ than in $\mathrm{D} 2 \mathrm{O}$, an effect attributed to hydrogen bond jumping events, which involve large-scale, rapid motion of the hydrogen or deuterium atom[4] and would therefore be particularly sensitive to isotope substitution.[3] If viewed as a barrier crossing event, the relaxation would be dominated by intrawell, local fluctuations where the hydrogen bond is preserved, with only a minor contribution from barrier crossings (i.e. angular jumps). The presence or lack of the observed isotope effect can be used to determine the extent to which hydrogen bond jumping events are suppressed near hydrophobic interfaces.

Previous work has shown that small hydrophobic solutes have been shown to have little influence on the hydration dynamics and conserve the $30 \%$ relaxation difference between $\mathrm{H} 2 \mathrm{O}$ and D2O.[3] Near the structured hydrophobic region of HEWL-RC, however, the isotope effect vanishes, despite the relaxation occurring on timescales indicative of water hydration (Figure 2b). This observation is consistent with the intra-well fluctuations not being hindered, but the angular jumping events being significantly stifled by the hydrophobic surface of the protein. The unfavorable orientation that water must adopt surrounding a hydrophobic surface, and the lack of hydrogen bonding opportunities on the protein surface, results in a suppression of hydrogen bond jumps.

The random coil portion of the protein offers a significantly different surface, defined less by a flat collective surface and more by individual residues, where hydration resembles that experienced by a small molecule.
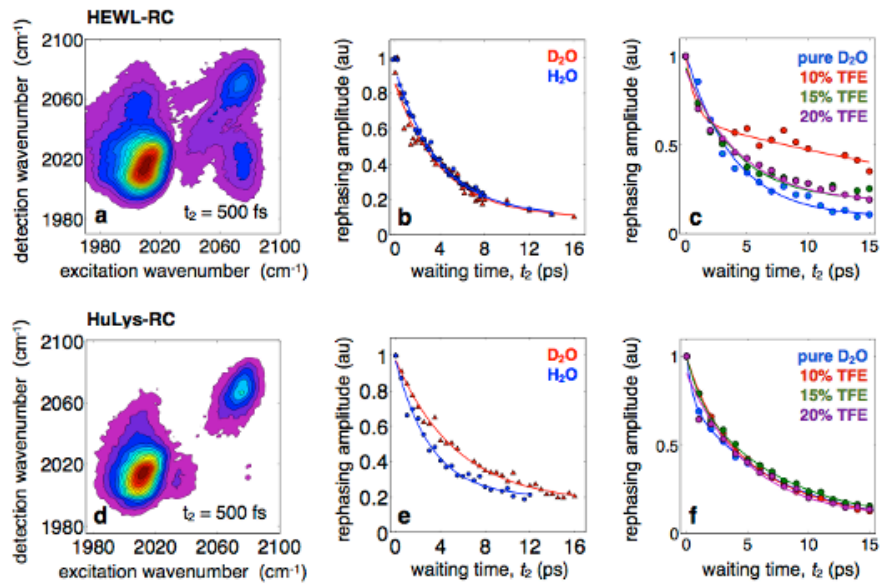

Fig. 2 2D-IR rephasing spectra of HEWL-RC (a) and HuLys-RC (d) shown at a waiting time of $500 \mathrm{fs}$. The low-frequency mode centered at $2004 \mathrm{~cm}^{-1}$ was used for analysis. Vibrational relaxation of the probe in $\mathrm{H}_{2} \mathrm{O}$ and $\mathrm{D}_{2} \mathrm{O}$ shows no isotope dependence for HEWL-RC (b), whereas it is $30 \%$ faster in $\mathrm{H}_{2} \mathrm{O}$ than in $\mathrm{D}_{2} \mathrm{O}$ for HuLys-RC (e). The location of the protein hydrated by constrained water also shows a susceptibility for dehydration by TFE cosolvent, evidenced by the large increase in lifetime at low concentrations of TFE (c). Likewise, the location of the protein hydrated by bulk-like water shows no dependence on TFE, remaining hydrated at all TFE concentrations (f). The \% TFE concentrations shown are volume percent.

Indeed, we observe a clear

isotope effect which strongly suggests that the hydration dynamics surrounding the random coil portion of HuLys-RC exhibits the bulk-like dynamics unconstrained water (Figure 2e).[3] These results demonstrate that the hydration environment of a globular protein is remarkably heterogeneous, extending over the full range from protein-mediated hydrogen bond jump suppression to fully liberated bulk solvation. Thus, the protein serves to influence the dynamics of the solvent by imposing its own surface heterogeneity, which is topologically-directed and sitespecific.

\section{Solvent exchange}

Many biological processes, like association reactions (i.e. protein-protein interactions) and preferential hydration, occur at the protein-water interface and tend to involve the dehydration of significant portions of the protein.[5] Heterogeneously constrained water around a globular protein provides a favorable thermodynamic reservoir to drive and direct such processes since dehydration is both enthalpically and entropically favorable. Here, we leverage a simple solvent exchange process, 
using 2,2,2-trifluoroethanol (TFE) as an amphiphilic cosolvent, to demonstrate the connection between constrained water and surface processes. Upon the addition of $10 \%$ TFE to a solution of HEWL-RC in D2O the vibrational lifetime increases by an order of magnitude (Figure 2c). The vibrational lifetime of HEWL-RC in $10 \%$ TFE $(32 \pm 2 \mathrm{ps})$ is nearly identical to the lifetimes observed for other metal-carbonyls in alcohol solvents. Thus, in the region of constrained water the hydration environment is exchanged for an alcohol environment. Further addition of TFE results in the denaturation of the protein, which can be observed in the return of the lifetime to timescales characteristic of water-assisted relaxation.[3]

It is also observed that the region of the protein hydrated by bulk-like water is insensitive to the addition of TFE (Figure 2f), showing clearly that this region is not dehydrated by the alcohol cosolvent even at elevated concentrations. In this region, there is no net thermodynamic driving force to liberate the already bulk-like water. These results demonstrate that the liberation of constrained water from the protein surface is necessary to provide a driving force for association reactions. Furthermore, the denaturation of proteins by TFE is shown to work through a direct mechanism, where hydrophobic regions of the proteins are dehydrated and the protein becomes partially solvated by alcohol solvent. Dehydrating a sufficient amount of water from the protein surface results in an insufficient hydrophobic pressure for the protein to remain in a compact folded state, and therefore the protein denatures.[6]

Experimental techniques that can directly access the dynamics occurring at the protein-water interface will prove valuable for gaining a full understanding of this key region. Here, we have presented the first step in vibrational labeling techniques that will allow for hydration dynamics around proteins to be studied directly, and at biologically relevant concentrations.

[1] P. Ball, Chem. Rev., 108, 74 (2008)

[2] D. Chandler Nature, 437, 640 (2005)

[3] J.T. King, M.R. Ross, K.J. Kubarych J. Phys. Chem. B, 116, 3754 (2012)

[4] D. Laage, J.T. Hynes Science, 311, 832 (2006)

[5] S.J. Wodak, J. Janin Adv. Protein Chem., 61, 9 (2002)

[6] J.F. Povey, C.M. Smales, S.J. Hassard, M.J. Howard J. Struct. Biol. 157, 329 (2007) 